\title{
FUNCIONES EJECUTIVAS, CONDUCTA SEXUAL DE RIESGO Y USO DE DROGAS LÍCITAS E ILÍCITAS EN ADOLESCENTES: UNA REVISIÓN SISTEMÁTICA
}

\section{EXECUTIVE FUNCTIONS, RISKY SEXUAL BEHAVIOUR AND ILICIT AND LICIT DRUG USE IN ADOLESCENTS: A SYSTEMATIC REVIEW}

Mayra Mendoza-Armenta', Carolina Valdez-Montero', Martha O. Valle-Solís², Jesica G. Ahumada-Cortez', Mario E. Gámez-Medina1

${ }^{1}$ Facultad de Enfermería Mochis, Universidad Autónoma de Sinaloa. México

${ }^{2}$ Unidad Académica de Enfermería, Universidad Autónoma de Nayarit. México

\section{Abstract}

Objective. To identify and synthesize the available evidence of the relationship between Executive Functions (EFs), sexual risk behavior and illicit and licit drug use in adolescents. Method. Search was performed on: Elsevier, Academic Search Complete (Ebsco), MedicLatina (Ebsco), Bussines Source Complete (Ebsco), Regional Business News (Ebsco) and Google Scholar. The DeCs and MeSH descriptors and the Joanna Briggs Institute checklist were used for study selection. Results. Eight studies were chosen. $62.5 \%$ of the studies mentioned the EFs in a general or some component(s). $37.5 \%$ of the studies showed that the lower EFs, the greater the sexual risk behavior and drug use. Conclusions. EFs are a complex construct and has an important role in the regulation of behavior, as well as in the decision-making and attention of adolescents. The findings indicate that EFs are related to sexual risk behaviors, however, a variety is found in the results regarding drug use.

Keywords: Adolescents, sexual risk behavior, executive functions, drug use.

\section{Resumen}

Objetivo. Identificar y sintetizar la evidencia disponible de la relación entre las Funciones Ejecutivas (FE), conducta sexual de riesgo y uso de drogas lícitas e ilícitas en adolescentes. Método. Se realizó búsqueda en: Elsevier, Academic Search Complete (Ebsco), MedicLatina (Ebsco), Bussines Source Complete (Ebsco), Regional Business News (Ebsco) y Google Académico. Se utilizó los descriptores DeCs y MeSH y la lista de chequeo de Joanna Briggs Institute para la selección de los estudios. Resultados. Se eligieron ocho estudios. El $62.5 \%$ de los estudios abordaron las FE de manera general o algún(os) componente(s). El 37.5\% de los estudios mostraron que a menor FE mayor conducta sexual de riesgo y uso de drogas. Conclusiones. Las FE es un constructo complejo y juega un papel importante en la regulación de la conducta, así como en la toma de decisiones y la atención de los adolescentes. Los hallazgos indican que las FE se relacionan con las conductas sexuales de riesgo, sin embargo, se encuentra una variedad en los resultados con respecto al consumo de drogas.

Palabras clave: Adolescentes, conducta sexual de riesgo, funciones ejecutivas, uso de drogas. 
Las Funciones Ejecutivas (FE) son un elemento fundamental en los procesos cognitivos. Estos originan las principales actividades psicológicas y las características de la conducta de los seres humanos (Barker et al., 2014). Según Yoldi (2015), los procesos de control de las FE se hallan localizados en la corteza prefrontal. Las FE implican procesos cerebrales complejos, por lo cual ha surgido la necesidad de identificar los componentes que la integran (Ferreira, Zanini, \& Seabra, 2015); sin embargo, existe una diversidad en cuanto a su clasificación (Toplak, West, \& Stanovich, 2012).

De acuerdo al modelo referido por Papazian, Alfonso y Luzondo (2006), las FE están constituidas por los siguientes componentes: memoria de trabajo, monitorización, flexibilidad, inhibición y planeación. Sin embargo, dentro de los componentes más reconocidos y estudiados en la literatura científica se encuentra el Control inhibitorio (Cinh), Memoria de Trabajo (MT) y la Flexibilidad Cognitiva (FC) (Diamond, 2013; Ferreira et al., 2015). El Cinh se define como el componente el cual dirige la atención, el trayecto del pensamiento, la conducta y las emociones. Este incluye dos tipos importantes de inhibición: el control de la interferencia y la inhibición de la respuesta. El primero es responsable de inhibir o enfocar el yo interior, mientras el segundo implica la supresión de comportamientos (Santa-Cruz \& Rosas, 2017). Mientras que la MT se refiere a un proceso mental que de acuerdo a la edad funciona para almacenar, monitorizar y manejar información. Y por último la FC, siendo la habilidad para crear formas de resolución de problemas (Santa-Cruz \& Rosas, 2017).

Las FE juega un papel importante en la toma de decisiones, principalmente en las etapas tempranas, como es en la adolescencia (Kuhn, 2006). Pharo, Slim, Graham, Gross \& Hayne (2011) señalan que los adolescentes son susceptibles a involucrarse en conductas de riesgo, siendo más vulnerables aquellos que presentan una personalidad impulsiva o un déficit neuropsicológico (Oliva \& Antolín, 2010). Según HuitrónBravo et al. (2011) y Alfonso \& Figueroa (2017) la mayoría de los problemas de salud en los adolescentes son consecuencia de conductas de riesgo, esto se debe a que todavía está en un progreso cognitivo de formulación de sus pensamientos, ideas y personalidad los cuales regulan su comportamiento sexual (Castillo-Arcos et al., 2017). Según Granados \& Sierra (2016) algunos de los riesgos sexuales durante la adolescencia son; los embarazos no deseados, adquirir Infecciones de Transmisión Sexual (ITS) y/o el Virus de Inmunodeficiencia Humana (VIH).

Otro de los comportamientos de riesgo que se presenta frecuentemente durante la adolescencia es el uso de drogas, conllevando a la afección de los múltiples sistemas neurobiológicos y con ello a los procesos cognitivos y conductuales (Mariño, Castro, \& Torrado, 2012). El uso de drogas puede provocar una disminución en la percepción del riesgo, una sobrevaloración de las capacidades y un sentimiento de invulnerabilidad (Alvarado, Lucero, \& Salinas, 2011). De acuerdo a lo anterior señalado, es importante conocer el funcionamiento neuropsicológico de los adolescentes ya que en esta etapa de la vida aún existen cambios cruciales en las áreas de la corteza prefrontal, lo cual puede conllevar que el adolescente tome decisiones de riesgo potencial comprometiendo su salud sexual. Por lo anterior, el objetivo del presente estudio es identificar y sintetizar la evidencia disponible de la relación entre las FE, la conducta sexual de riesgo y el uso de drogas lícitas e ilícitas en adolescentes.

\section{MÉTODO}

La presente revisión sistemática se realizó de acuerdo con las 5 etapas sugeridas por Ferreira, Urrutia y Alonso-Coello (2011): 1) Definición de la pregunta clínica de interés y los criterios de inclusión y exclusión de los estudios; 2) Localización y selección de los estudios relevantes; 3) Extracción de datos de los estudios primarios; 4) Análisis y presentación de los resultados y 5) Interpretación de los resultados.

Para guiar la presente revisión sistemática, se formuló la siguiente pregunta: ¿Cuál es la evidencia científica existente sobre la relación entre las FE, la conducta sexual de riesgo y el uso de drogas lícitas e ilícitas en los adolescentes?

En lo que respecta a los criterios de inclusión de los artículos científicos primarios, se consideró aquellos que abordaran la variable de las FE o algún(os) componente(s) y que mostraran relación con la conducta sexual de riesgo y/o con el uso de drogas. Se consideraron publicaciones del año 2011 al 2018, contemplando estudios descriptivos-correlacionales, de cohorte de tipo transversal y longitudinal. Los estudios debían incluir a 
participantes adolescentes, de ambos sexos, cualquier nacionalidad, escolarizados y no escolarizados. Los criterios de exclusión fueron: que solo abordara una variable de interés, que se trataran de informes de casos informales, capítulos de libros, disertaciones, tesis, informes, noticias, editoriales, textos no científicos, y que no cumplieran con la calidad metodológica evaluada por la lista de chequeo de Joanna Briggs Institute (JBI, 2017).

Para localizar los estudios se realizó la búsqueda en las bases de datos: Elsevier, Academic Search Complete (Ebsco), Mediclatina (Ebsco), Bussines Source Complete (Ebsco), Regional Business News (Ebsco), se recurrió al buscador Google Académico para localizar artículos que no aparecieron en texto completo en las bases de datos. Se seleccionaron estudios de diversas disciplinas de la salud (medicina, psicología y psiquiatría), disciplinas que con mayor frecuencia estudian el fenómeno de interés. La primera búsqueda se realizó en marzo de 2018 y se Ilevó a cabo una segunda búsqueda en abril de 2018, con la finalidad de ampliar el número de artículos. Se utilizaron palabras claves, descriptores DeCS, Mesh y operadores booleanos, se realizaron diferentes combinaciones para garantizar una búsqueda amplia (Tabla 1). En lo que concierne a los criterios de selección de los estudios fueron basados en lista de Joanna Briggs Institute (JBI, 2017), evaluando los siguientes puntos: 1) Resumen estructurado; 2) Diseño metodológico claro y conciso; 3) Participantes del estudio fueron muestreados de manera apropiada; 4) Tamaño de la muestra adecuado; 5) Descripción a detalle de los participantes; 6) Instrumentos validados; 7) Análisis estadístico apropiado y 7) Resultados congruentes con los objetivos.

Tabla 1. Combinaciones para la búsqueda de literatura

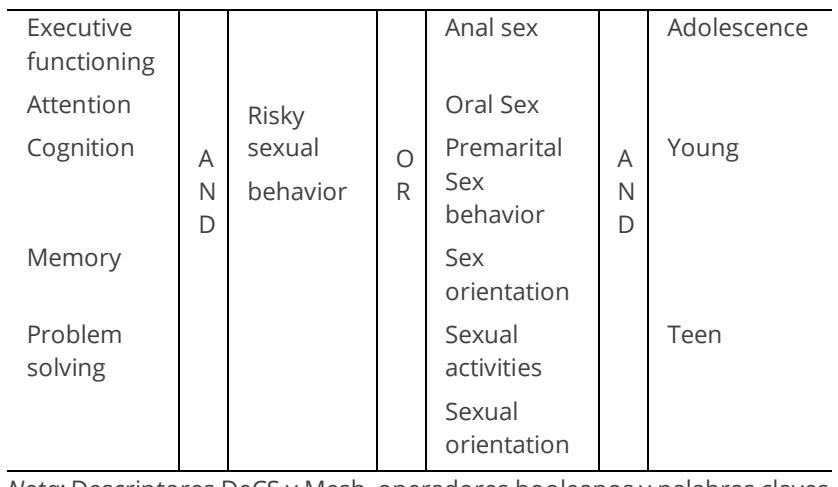

Nota: Descriptores DeCS y Mesh, operadores booleanos y palabras claves.

Para seleccionar la información de los estudios primarios se utilizó el formato de extracción de datos de revisiones sistemáticas de etiología y riesgo (JBI, 2019).
Los aspectos extraídos de cada uno de los estudios deben incluir detalles específicos sobre los adolescentes, la exposición de interés y los resultados de importancia para la pregunta planteada en el estudio. Se consideró los siguientes apartados: Autor, año, revista, métodos (diseño del estudio, entorno, características de los participantes, procedimiento del reclutamiento, duración del estudio o seguimiento de los participantes). Asimismo, se consideraron los resultados de las mediciones de las variables de las FE, la conducta sexual de riesgo y el uso de drogas.

En cuanto al análisis, presentación e interpretación de los resultados, se utilizó una síntesis de resumen narrativos para presentar los datos extraídos de los estudios individuales, asimismo, se realizó una tabulación de los datos (JBI, 2019), realizando una agrupación de los estudios de acuerdo con la variable de riesgo (conducta sexual de riesgo, uso de drogas). Para la presentación de los resultados se consideró la descripción de los estudios, características de los estudios, descripción de las variables; FE, conducta sexual de riesgo y uso de drogas y finalmente se presenta la relación entre las variables.

\section{RESULTADOS}

\section{Descripción de los estudios}

Inicialmente se encontraron 369 estudios potenciales de elegibilidad. Después de eliminar los duplicados, se recuperaron 313 para su posterior revisión, se excluyeron artículos que no cumplían con los criterios de inclusión establecidos, ni con los requisitos de la pregunta de investigación, arrojando un total de 14 artículos para evaluación de la calidad metodológica, de los cuales se eliminaron seis por no cumplir con el rigor metodológico indicado, obteniendo ocho estudios que reúnen los requerimientos para ser parte de la revisión sistemática (Figura 1).

\section{Características de los estudios}

De los estudios incluidos, el $62.5 \%$ fueron descriptivo correlacional, $25 \%$ de cohorte longitudinal y $12.5 \%$ comparativo transversal. El 50\% de los estudios es de tipo transversal y el otro $50 \%$ es de tipo longitudinal. Los lugares donde se llevaron a cabo los estudios fueron en áreas urbanas y rurales, entornos clínicos, 
instituciones educativas (secundarias) y albergues para jóvenes sin hogar. El intervalo de edad de los participantes osciló entre los 10 a los 24 años, en lo que respecta al género se identificó que el $87.5 \%$ estudió a masculinos y femeninos, y solo un $12.5 \%$ abordó a hombres homosexuales y/o bisexuales. Se identificó que el $62.5 \%$ de los estudios fueron realizados en Estados Unidos de América, 25\% en Holanda y $12.5 \%$ en Nueva Zelanda. El tamaño de la muestra fue diverso, esto puede deberse al tipo de diseño, ya que en los estudios de tipo transversal se identificaron muestras más pequeñas en comparación con los estudios longitudinales.

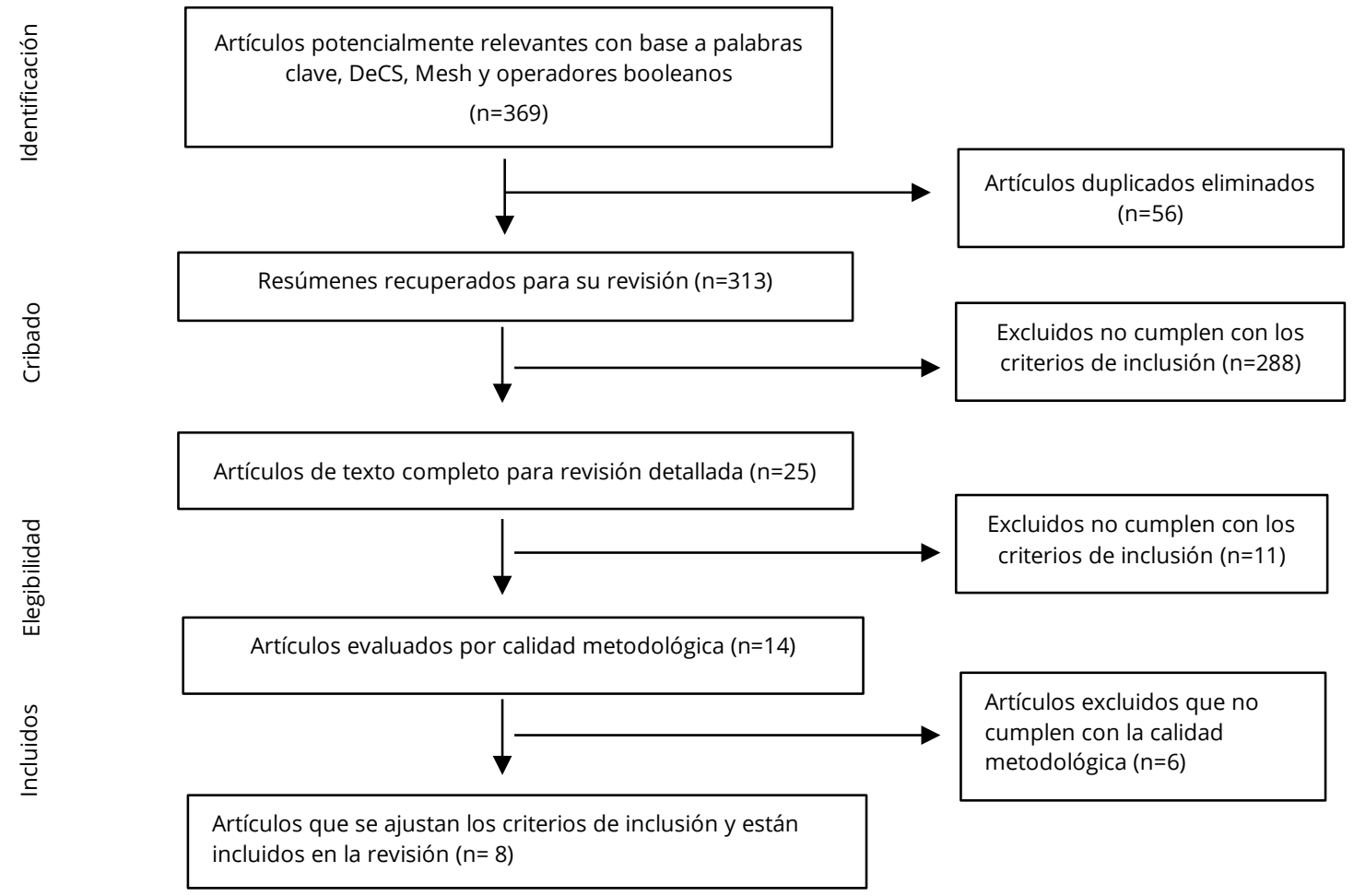

Figura 1. Selección de los estudios (Moher, Liberati, Tetzlaff, \& Altman, 2009)

Para la clasificación del nivel de evidencia de los artículos, se utilizó la categorización de la Agency for Healthcare Research and Quality (AHRQ). El nivel de evidencia que prevaleció (75\%) fue el IV, que corresponde a estudios con diseño no experimental e investigación correlacional, y posteriormente el nivel III (25\%), el cual se refiere a estudios con un diseño cuasi-experimental, como estudios sin aleatorización con un solo grupo previo y posterior a la prueba, serie temporal o control de casos (Tabla 2). El $62.5 \%$ de los estudios mencionan haberse adherido a las normas éticas de comités institucionales que rigen la investigación, y el 37.5\% no menciona los aspectos éticos para la realización del estudio. El 100\% de los estudios refiere tener ausencia de conflictos de intereses. En lo que respecta a las fechas de publicación, cuatro (50\%) de los artículos fueron publicados en el periodo $2015-2018$ y el otro $50 \%$ de los estudios fueron publicados en el periodo 2011-2012. Las revistas en las que fueron publicadas los estudios presentaron Factor de Impacto (FI) entre 0.20 a 4.815.

\section{Funciones ejecutivas}

En los ocho artículos se evaluó las FE. El 62.5\% de los artículos estudió las FE de manera general considerando varios componentes como; Cinh, MT, FC, cambio de atención y atención sostenida, resolución de problemas, utilizando baterías neuropsicológicas (Boelema et al., 2015; Golub, Starks, Kowalczyk, Thomposn, \& Parsons 2012; Hanson, Lisdahl, Padula, Tapert, \& Brown 2011; Pharo et al., 2011; Piche, Kaylegian, Smith, \& Hunter, 2018). Mientras que el $37.5 \%$ evaluó uno o dos de los componentes principales de las FE (Kim-Spoon et al., 2016; Peeters et al., 2015; Romer et al., 2011). 
Tabla 2. Características y resumen de los estudios incluidos

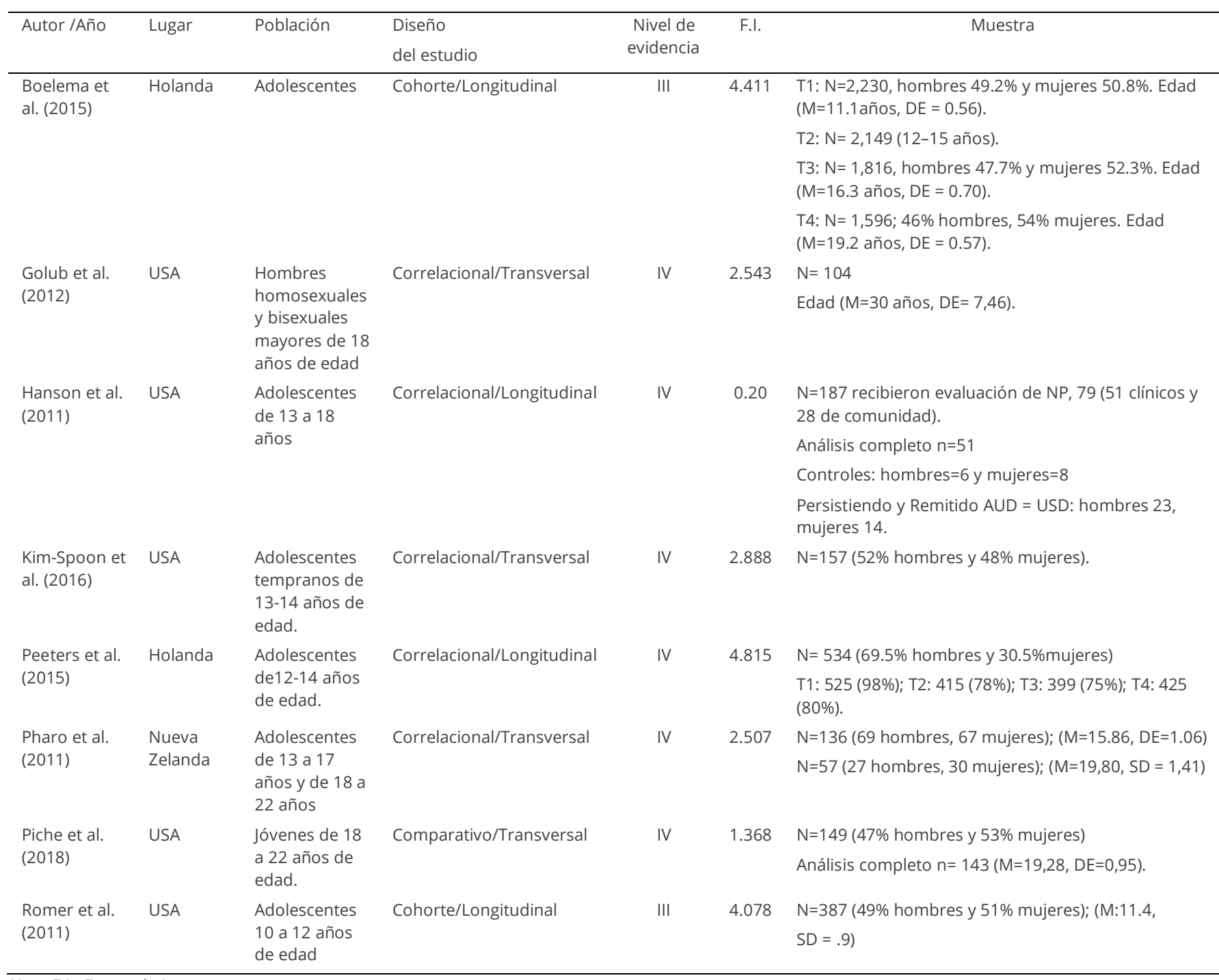

Nota: F.I.=Factor de Impacto.

Se identificó en los estudios analizados algunas pruebas neuropsicológicas que se usaron con mayor frecuencia para la medición de las FE o alguno de sus componentes, Hanson et al. (2011), Pharo et al. (2011) y Romer et al. (2011) utilizaron la tarea Digit Span la cual es una prueba que evalúa la memoria de trabajo y aspectos auditivos-verbales. Hanson et al. (2011), Pharo et al. (2011) y Piche et al. (2018) midieron con la prueba WAIS la cual permite evaluar el vocabulario, similitudes, diseño de bloques, aritmética y algunas subpruebas de la prueba de Digit Span. En los estudios de Hanson et al. (2011) y Pharo et al. (2011) se utilizó la prueba COWAT que mide la fluidez verbal, velocidad de procesamiento y capacidad de inhibición, planificación y organización, y el WMS instrumento diseñado para evaluar los aspectos principales del funcionamiento de la memoria, permitiendo evaluar la capacidad de almacenar y recuperar de forma consciente aspectos concretos de información referida a una situación o contexto específico y la memoria de trabajo. Los estudios de Hanson et al. (2011) y Piche et al. (2019) utilizaron la prueba de CVLT la cual evalúa la memoria verbal y la capacidad de aprendizaje, la medida de Stroop fue utilizada por Peeters et al. (2015) la cual permite valorar aspectos como la atención selectiva y de control inhibitorio, y Golub et al. (2012) y Piche et al. (2018) midieron con la IGT la cual permite realizar una prueba de juicio y toma de decisiones diseñada para identificar a pacientes con lesiones de la corteza prefrontal, que demuestra consistentemente sensibilidad a los déficits neurocognitivos entre individuos dependientes de sustancias psicoactivas (Tabla 3). 
Tabla 3. Instrumentos neuropsicológicos por autores

\begin{tabular}{|c|c|c|c|c|c|c|c|c|}
\hline & $\begin{array}{c}\text { Boelema et } \\
\text { al.(2015) }\end{array}$ & $\begin{array}{c}\text { Golub et al. } \\
\text { (2012) }\end{array}$ & $\begin{array}{c}\text { Hanson et al. } \\
\text { (2011) }\end{array}$ & $\begin{array}{c}\text { Kim-Spoon et } \\
\text { al. (2016) }\end{array}$ & $\begin{array}{c}\text { Peeters } \\
\text { et al. } \\
(2015)\end{array}$ & $\begin{array}{c}\text { Pharo et al. } \\
\text { (2011) }\end{array}$ & $\begin{array}{c}\text { Piche et al. } \\
\text { (2018) }\end{array}$ & $\begin{array}{c}\text { Romer et al. } \\
\text { (2011) }\end{array}$ \\
\hline ANT & * & & & & & & & \\
\hline WCST & & * & & & & * & & \\
\hline Counting Span & & * & & & & & & \\
\hline WISC-R & & & * & & & & & \\
\hline Digit Span & & & * & & & * & & * \\
\hline COWAT & & & * & & & * & & \\
\hline WMS & & & * & & & * & & \\
\hline CVLT & & & * & & & & * & \\
\hline WAIS & & & * & & & * & * & \\
\hline BIS/BAS & & & & * & & & & \\
\hline SOPT & & & & & * & & & \\
\hline Stroop & & & & & * & * & & \\
\hline Mental control & & & & & & * & & \\
\hline ATQ & & & & & & & * & \\
\hline $\begin{array}{l}\text { BRIEF (IM, BRI, } \\
\text { GEC) }\end{array}$ & & & & & & & * & \\
\hline D-KEFS & & & & & & & * & \\
\hline ITG o variante & & * & & & & & * & \\
\hline TOL & & & & & & & * & \\
\hline WAI & & & & & & & * & \\
\hline WARML2 & & & & & & & * & \\
\hline MSIT & & & & * & & & & \\
\hline CANTAB & & & & & & & & * \\
\hline Letter two back & & & & & & & & * \\
\hline $\begin{array}{l}\text { Sensation Seeking } \\
\text { Scale }\end{array}$ & & & & & & & & * \\
\hline Go/No-Go & & * & & & & & & \\
\hline
\end{tabular}

Nota: *Utilizó instrumento; ANT= Amsterdam Neuropsychological Task; WCST= Wisconsin Cart Sort Test; WISC-R= Escala de Inteligencia Wechsler; COWAT= Test de asociación de palabras orales controladas; WMS= Escala de Memoria de Wechsler; CVLT= California Verbal Learning Test; WAIS= Wechsler Adult Intelligence Scale-Revised; BIS/BAS= Sistema de comportamiento de inhibición y sistema de enfoque de comportamiento; SPOT= Self Ordered Pointing Task, ATQ= Adult Temperament Questionnaires; BRIEF= Inventario de Calificación de Comportamiento de la Función Ejecutiva; IM= Indice de Metacognición; BRI= Indice de evaluación de comportamiento; GEC=Indice Global Executive Composite; D-KEFS= Delis-Kaplan Executive Functioning; IGT= Iowa Gambling Task; TOL= Torre de Londres; WAI=Weinberger Inventory; WARML2= Wide Range Assement of Memory and Learning; MSIT= Multi-Source Interference Task; CANTAB $=$ tarea informatizada auto-dirigida; Go/Nogo= Ir o no ir.

\section{Conducta sexual de riesgo}

En lo que respecta a la variable de conducta sexual de riesgo se logró identificar que el 37.5\% de los artículos la abordaron (Golub et al., 2012; Pharo et al., 2011, \& Piche et al., 2018), cabe destacar que los estudios utilizan instrumentos que miden de manera general las conductas de riesgo, entre ellas, la conducta sexual. Pharo et al. (2011) encontró que los hombres y los adolescentes de mayor edad tenían puntuaciones más altas en relación con la frecuencia de las relaciones sexuales, múltiples parejas sexuales y no usar condón. A su vez, Golub et al. (2012) demostró que los participantes reportaron actos sexuales de alto riesgo, tales como sexo anal sin protección con una pareja ocasional o pareja principal serodiscordante, relaciones sexuales bajo la influencia de drogas, e identificaron una media de 12 actos sexuales anales en los últimos 30 días. Por su parte Piche et al. (2018) evaluaron el número de parejas sexuales que han tenido en su vida, considerando siete niveles, que va desde cero a más de seis parejas sexuales, el estudio demostró que los adolescentes han tenido en promedio cuatro parejas sexuales en su vida.

\section{Uso de drogas}

En lo que respecta a la variable de uso de drogas se identificó que el $100 \%$ de los artículos la abordan. Peeters et al. (2015) demostraron que los participantes de educación especial presentaron medias más altas en la 
cantidad de consumo de alcohol en comparación con los participantes que se encontraban en un ambiente educativo regular. Por su parte Boelema et al. (2015) descubrieron que los bebedores crónicos de alcohol tuvieron una mayor prevalencia de consumo de alcohol, tabaco y cannabis en comparación con los no bebedores o bebedores pocos frecuentes. Asimismo, el estudio demostró que los bebedores crónicos tuvieron mayores puntuaciones respecto al consumo de alcohol materno y paterno, siendo aún mayor en el consumo paterno.

El estudio de Golub et al. (2012) identificaron que los participantes reportaron un promedio de 9.94 días de consumo de drogas en los últimos 30 días y que casi la mitad de los participantes presentaron uso problemático de drogas. Por su parte, el estudio de Hanson et al. (2011) demostró que al examinar varios indicadores del uso de sustancias como el alcohol, drogas ilegales y drogas médicas y los síntomas promedio de abstinencia de drogas durante los 10 años pareció predecir las habilidades en los participantes en la edad adulta. A su vez, el estudio de Kim-Spoon et al. (2016) descubrieron presencia del uso severo de cigarrillo, alcohol y cannabis en adolescentes. Pharo et al. (2011) en su estudio demostró que los hombres tuvieron mayor consumo de tabaco, drogas y alcohol en comparación con las mujeres, y los adolescentes de menor edad reportaron mayor consumo de tabaco en comparación con los de mayor edad. El estudio de Piche et al. (2018) demostró que los adolescentes tienen presencia de abuso de alcohol y dependencia, siendo mayor en el abuso de alcohol. Mientras Romer et al. (2011) identificaron un incremento en el consumo de alcohol y cigarrillos en adolescentes.

\section{Relación entre las variables de las FE, conducta sexual de riesgo y uso de drogas.}

De acuerdo con los datos obtenidos de los estudios, se identificó que el $37.5 \%$ mostró una relación entre las FE y la conducta sexual de riesgo (Golub et al., 2012; Pharo et al., 2011; Piche et al., 2018). En el estudio de Golub et al. (2012) se identificó una relación entre los subtipos de las FE con actos sexuales de riesgo, tales como: tener relaciones sexuales sin uso de condón con una pareja ocasional o pareja estable y haber llevado a cabo relaciones sexuales bajo efecto de alguna droga. A su vez, Pharo et al. (2011) refieren que las pruebas neuropsicológicas para la medición de las FE se relacionaron negativamente con la frecuencia de las relaciones sexuales, las múltiples parejas sexuales y el no usar el condón. Mientras que Piche et al. (2018) refieren que los jóvenes con niveles bajos de las FE tienen mayor número de parejas sexuales.

El 100\% de los estudios indagaron sobre la relación entre las FE y el uso de drogas, de los cuales el $75 \%$ identificaron una relación significativa (Golub et al., 2012; Hanson et al., 2011; Kim-Spoon et al., 2016; Pharo et al., 2011; Piche et al., 2018; Romer et al., 2011). Golub et al. (2012) demostraron una relación entre las FE de alto rendimiento, bajo rendimiento y bajo desempeño, con el uso de cocaína, metanfetamina y éxtasis. En la investigación realizada por Hanson et al. (2011) los participantes que tenían una baja FE presentaron un consumo más temprano de alcohol, drogas ilegales y drogas médicas. Mientras tanto, Kim-Spoon et al. (2016) refieren que el proceso de recompensa (elemento de la FE) se relacionó con el inicio temprano de sustancias y el uso severo de cigarrillo, alcohol y marihuana.

En cuanto al estudio de Pharo et al. (2011) los participantes que puntuaron más bajo en la batería neuropsicológica mostraron relación con el consumo de drogas lícitas e ilícitas, comparado con los participantes que obtuvieron puntuaciones más altas. Por otro lado, Piche et al. (2018) refieren que los jóvenes sin hogar con un nivel bajo de las FE se involucran más fácilmente en conductas de riesgo relacionadas con el consumo de drogas, tales como; el alcohol, marihuana y otras drogas y Romer et al. (2011) refieren una relación entre la presencia de un déficit de la memoria de trabajo y el consumo de alcohol y tabaco.

El 25\% de los estudios indicaron que no existe relación entre las variables de las FE y uso de drogas (Boelema et al., 2015; Peeters et al., 2015). En la investigación de Boelema et al. (2015) se identificó que entre los grupos de bebedores de alcohol no revelaron diferencias significativas en la maduración del funcionamiento ejecutivo. Asimismo, el estudio de Peeters et al. (2015) demostró que la asociación entre las FE y el consumo de alcohol no puede interpretarse como un efecto del consumo de alcohol. Cabe destacar que el 37.5\% de los estudios (Golub et al. 2012; Pharo et al., 2011; Piche et al., 2018) mostraron que a menor funcionamiento ejecutivo mayor conducta sexual de riesgo y uso de drogas (Tabla 4). 
Tabla 4. Relación de las funciones ejecutivas con conducta sexual de riesgo y uso de drogas por autores

\begin{tabular}{lcc}
\hline & $\begin{array}{c}\text { Conducta } \\
\text { sexual de } \\
\text { riesgo }\end{array}$ & $\begin{array}{c}\text { Uso de } \\
\text { drogas }\end{array}$ \\
\hline Boelema et al. (2015) & X & NRS \\
Golub et al. (2012) & RS & RS \\
Hanson et al. (2011) & $X$ & RS \\
Kim-Spoon et al. (2016) & X & RS \\
Peeters et al. (2015) & RS & RS \\
Pharo et al. (2011) & RS & RS \\
Piche et al. (2018) & X & RS \\
Romer et al. (2011) & R
\end{tabular}

Nota: RS= Relación Significativa; NRS= No Relación Significativa; X= No se estudia la variable.

\section{DISCUSIÓN}

El objetivo de la presente revisión sistemática fue identificar y sintetizar la evidencia disponible de la relación entre las $\mathrm{FE}$, la conducta sexual de riesgo y el uso de drogas lícitas e ilícitas en adolescentes. En lo que respecta a la metodología utilizada, todos los artículos estudiaron las FE o alguno(s) de sus componentes mediante el uso de pruebas neuropsicológicas o un conjunto de estas definidas como baterías. Si bien, existen variados instrumentos validados para la evaluación de las FE, de acuerdo con un análisis profundo se derivó que las pruebas neuropsicológicas tan sólo permiten evaluar ciertos componentes, no existiendo actualmente un instrumento completo que permita la medición de todos los componentes que integran las FE (Buller, 2010). Según Villegas-Pantoja, Alonso-Castillo, Benavides-Torres \& Guzmán-Facundo (2013) las FE se compone como un apartado unitario o de diversos componentes independientes pero interconectados entre sí. Asimismo, se identificó que los elementos de las FE más estudiados fueron el Cinh, MT y la FC, este hallazgo concuerda con diversos estudios, los cuales consideran estos componentes como los principales (Blair, 2016; Diamond, 2013; Santa \& Rosas, 2017).

En lo que respecta a las variables de conducta sexual de riesgo y el uso de drogas se utilizaron cuestionarios autoaplicados y entrevistas individuales, los primeros constituyen un método adecuado y directo en la evaluación de las respuestas cognitivas y experiencia subjetiva del individuo (Catresana \& Revuelta, 1992), mientras que la entrevista, según Díaz-Bravo, Torruco-
García, Martínez-Hernández \& Varela-Ruiz (2013), es una técnica que permite al entrevistador recabar datos más profundos de una o varias variables de interés. Referente a la conducta sexual de riesgo solo tres artículos la estudiaron, de los cuales utilizaron cuestionarios que medían conductas de riesgo incluyendo los riesgos sexuales, identificando que los adolescentes se ven inmersos en distintos comportamientos sexuales de riesgo que comprometen su salud sexual. El estudio de Rodríguez \& Traverso (2012) menciona que pudiera deberse a que en la etapa de la adolescencia aumenta la experimentación sexual y hay cambios imprevisibles de conducta, así como sentimientos de invulnerabilidad lo cual conlleva a que los adolescentes realicen conductas sexuales con mayor precocidad.

Por otra parte, todos los artículos abordaron la variable de uso de drogas, identificando que las sustancias más estudiadas fueron: alcohol, tabaco y cannabis. Este hallazgo concuerda con varios estudios (Font-Mayolas et al., 2013; Gárciga-Ortega, Torres, \& Jorge, 2015; Maturana, 2011; Tena-Suck, Castro-Martínez, Marín-Navarrete, Gómez-Romero, \& Gómez-Martínez 2018). Según Ramos-Valverde \& Moreno-Rodríguez (2010) existe evidencia de la relación progresiva en el consumo de las diferentes sustancias, de modo que el consumo de drogas lícitas (alcohol y tabaco) influye en el inicio del consumo de drogas ilícitas (cannabis), siendo esta última la droga más usada en todo el mundo (Galván, Guerrero-Martelo, Torres, Torres, \& Sánchez 2015). Uno de los factores que influye en el uso de drogas es la facilidad de acceso, favoreciendo así el consumo de estas (Iglesias, Cavada, Silva, \& Cáceres, 2007).

Otro hallazgo que se presentó fue que ante una FE baja mayor es la conducta sexual de riesgo; entre ellas la frecuencia de relaciones sexuales, relaciones sin uso de condón y múltiples parejas sexuales. El estudio de ValleSolís (2011) menciona que los adolescentes con bajas FE presentan mayores motivos para tener sexo, bajo control para sexo seguro y conducta sexual de riesgo, lo que indica que el proceso mental y de maduración en los adolescentes influye en la toma de decisiones sobre las relaciones sexuales, ya sean de riesgo o seguras. Por lo que, ante la presencia de un déficit de maduración de las $\mathrm{FE}$, mayor posibilidad de aumento de actividades sexuales de riesgo. 
Referente a la relación entre las FE y el uso de drogas, se identificó que más de la mitad de los estudios demostraron relación. De acuerdo con lo anterior el hallazgo es que los adolescentes que presentan menor funcionamiento ejecutivo mayor es el uso de drogas, tanto lícitas como ilícitas. Actualmente el uso de drogas se ha asociado con algunos cambios o déficits neuronales, lo cual conlleva evidenciar una relación entre los consumidores de drogas y el deterioro de las FE (Mariño et al., 2012). El uso de drogas ha sido asociado consistentemente con diversos problemas de salud, que tienen en común una alteración en las FE las cuales regulan la conducta, incluyendo la memoria, la atención y la toma de decisiones (Torres \& Fiestas, 2012). El estudio realizado por Bajo-Bretón (2011) identificó la presencia de un déficit en las FE específicamente en la MT y el Cinh en sujetos con consumo de alcohol y/o cannabis. Los hallazgos sugeridos anteriormente concuerdan con Viejo \& Ortega-Ruiz (2015) los cuales refieren que las FE juegan un papel importante en los comportamientos de riesgos y la toma de decisiones, lo que conlleva a que la inmadurez prefrontal favorece la implicación de los adolescentes en conductas sexuales de riesgo y el uso de drogas.

Es importante destacar que una cuarta parte de los estudios no presentó relación entre las FE y el uso de drogas. Esto puede deberse a la falta de unificación de los componentes de las FE lo que conlleva a la diversidad de resultados (Villegas-Pantoja et al., 2013). También pudiera deberse a que actualmente las técnicas de neuroimagen han aportado datos que indican que otras estructuras cerebrales desempeñan un importante papel en la conducta de riesgo como en el caso del uso de drogas en adolescentes (Oliva \& Antolín, 2010).

\section{Conclusión}

La presente revisión sistemática es una manera de recabar y sintetizar la mayor evidencia que exprese la situación de investigación y los posibles vacíos de conocimientos en el estudio de las FE, la conducta sexual de riesgo y el uso de drogas en adolescentes. De acuerdo a la información sintetizada de los artículos analizados, se llega a la conclusión de que las FE es un constructo complejo y que juega un papel importante en la regulación de la conducta, así como en la toma de decisiones y la atención de los adolescentes. Dicho constructo presenta una diversidad de componentes, lo que dificulta su medición y por ende la unificación de los resultados. Los componentes abordados con mayor frecuencia en los estudios fueron el control inhibitorio, memoria de trabajo y la flexibilidad cognitiva.

Con respecto a la conducta sexual de riesgo se concluye que los adolescentes frecuentemente se ven inmersos en actividades sexuales de riesgo; como múltiples parejas sexuales, no uso de condón, sexo anal sin protección y relaciones sexuales bajo la influencia de drogas, comprometiendo así su salud sexual. En cuanto al consumo de drogas, el hallazgo de los estudios seleccionados, se identificó que, las drogas más consumidas fueron el alcohol, el tabaco y la marihuana.

También se concluye que los estudios demostraron que a menor FE mayor es la conducta sexual y uso de drogas en los adolescentes.

Es importante señalar que dicho fenómeno repercute seriamente en la salud de los adolescentes, por lo que es importante considerarlo como una necesidad de atención para los profesionales de la salud. Se recomienda aplicar dentro del primer nivel de atención pruebas neuropsicológicas como complemento para la identificación de diagnósticos que conlleven a la prevención o tratamiento de problemas conductuales que comprometen al adolescente a llevar a cabo conductas de riesgo como es el caso de las conductas sexuales o el uso de drogas. Asimismo, se pudiera desarrollar o adaptar intervenciones interdisciplinarias dirigidas a los adolescentes, que permitan mejorar la capacidad cognitiva y toma de decisiones. Sin embargo, la presente revisión cuenta con algunas limitaciones, una de ellas fue la escasez de estudios incluidos en la revisión sistemática, pudiera ser por el uso de pocas bases de datos. Otra limitación fue que se realizaron búsquedas solo en dos idiomas (inglés y español), por lo que pueden existir más estudios con las variables de interés en otros idiomas.

\section{Agradecimientos}

Al Consejo Nacional de Ciencia y Tecnología (CONACyT), el trabajo fue apoyado por la beca nacional de posgrados (PNPC: 005560) otorgado a Mayra Mendoza Armenta 


\section{REFERENCIAS}

Alfonso, F. L., \& Figueroa, P. L. (2017). Conductas sexuales de riesgo en adolescentes desde el contexto cubano. Revista de Ciencias Médicas de Pinar del Río, 21(2), 143-151.

Alvarado, J. I. U., Lucero, J. C. V., \& Salinas, X. Z. (2011). Relación entre percepción de riesgo y consumo de drogas en estudiantes de bachillerato. Psicología y Salud, 21(1), 47-55. doi:10.25009/pys.v21i1.586

Bajo-Bretón, R. (2011). Deterioro cognitivo asociado al consumo de diferentes sustancias psicoactivas. Actas Españolas de Psiquiatría, 39(3), 168-73.

Barker, J. E., Semenov, A. D., Michaelson, L., Provan, L. S., Snyder, H. R., \& Munakata, Y. (2014). Less-structured time in children's daily lives predicts self-directed executive functioning. Frontiers in psychology, 5, 593. doi:10.3389/fpsyg.2014.00593

Blair, C. (2016). Developmental and ejecutive function. Association for psychological science, 25(1), 3-7. doi:10.1177/0963721415622634

Boelema, S. R., Harakeh, Z., van Zandvoort, M. J., Reijneveld, S. A., Verhulst, F. C., Ormel, J., \& Vollebergh, W. A. (2015). Adolescent heavy drinking does not affect maturation of basic executive functioning: longitudinal findings from the TRAILS study. PLoS One, 10(10). doi:10.1371/journal.pone.0139186

Buller, I. (2010). Evaluación neuropsicológica efectiva de la función ejecutiva. Propuesta de compilación de pruebas neuropsicológicas para la evaluación del funcionamiento ejecutivo. Cuadernos de Neuropsicología/Panamerican Journal of Neuropsychology, 4(1), 63-86.

Catresana, C. D. L. C. Y., \& Revuelta, J. G. D. R. Y. (1992). Autoinformes y respuestas sesgadas. Anales de Psiquiatría, 8(9), 362-366.

Diamond, A. (2013). Executive functions. Annual review of psychology, 64, 135-168. doi:10.1146/annurev-psych-113011-143750

Díaz-Bravo, L., Torruco-García, U., Martínez-Hernández, M., \& Varela-Ruiz, M. (2013). La entrevista, recurso flexible y dinámico. Investigación en educación médica, 2(7), 162-167.

Ferreira, G. I., Urrutia, G., \& Alonso-Coello. (2011). Revisiones sistemáticas y metaanálisis: bases conceptuales e interpretación. Revista Española de Cardiología. 64, 688-696. doi:10.1016/j.recesp.2011.03.029

Ferreira, L. de O., Zanini, D. S., \& Seabra, A. G. (2015). Executive Functions: Influence of Sex, Age and Its Relationship With Intelligence. Paidéia (Ribeirão Preto), 25, 383-391. doi:10.1590/1982-43272562201512

Font-Mayolas, S., Gras, M. E., Cebrián, N., Salamó, A., Planes, M., \& Sullman, M. J. M. (2013). Types of polydrug use among Spanish adolescents. Addictive Behaviors, 38, 1605- 1609. doi:10.1016/j.addbeh.2012.09.007

Galván, G., Guerrero-Martelo, M., Torres, J., Torres, F., \& Sánchez, A. (2015). Prevalencia de uso de cannabis: perfil familiar y social de una muestra adolescente. Psychologia: Avances de la Disciplina, 9(2), 101 112.
Gárciga-Ortega, O., Torres, C. S., \& Jorge, R. R. (2015). Consumo de drogas legales y estilo de vida en estudiantes de medicina. Revista Cubana de Salud Pública, 41(1), 4-17.

Golub, S. A., Starks, T. J., Kowalczyk, W. J., Thompson, L. I., \& Parsons, J. T. (2012). Profiles of executive functioning: Associations with substance dependence and risky sexual behavior. Psychology of Addictive Behaviors, 26, 895-905. doi:10.1037/a0029034

Granados, M. Reina, \& Sierra, Juan Carlos. (2016). Excitación sexual: una revisión sobre su relación con las conductas sexuales de riesgo. Terapia psicológica, 34(1), 59-70. doi:10.4067/S0718$\underline{48082016000100007}$

Hanson, K. L., Medina, K. L., Padula, C. B., Tapert, S. F., \& Brown, S. A. (2011) Impact of adolescent alcohol and drug use on neuropsychological functioning in young adulthood: 10-year outcomes. Journal of child \& adolescent substance abuse, 20(2), 135-154 doi:10.1080/1067828X.2011.555272

Huitrón-Bravo, G., Denova-Gutiérrez, E., Halley-Castillo, E, SantanderRigollet, S., Bórquez-Puga, M, Zapata-Pérez, L., Huinca, B., Zubarew, T. G., \& Villarroel-del-Pino, L. (2011). Conductas de riesgo en una muestra de adolescentes chilenos y mexicanos: un estudio comparativo. Papeles de población, 17(70), 33-47.

Iglesias, V., Cavada, G., Silva, C., \& Cáceres, D. (2007). Consumo precoz de tabaco y alcohol como factores modificadores del riesgo de uso de marihuana. Revista de Saúde Pública, 41, 517-522.

Joanna Briggs Institute Reviewer's Manual (2019). Data extraction. Recuperado de https://wiki.joannabriggs.org/display/MANUAL/7.3.6.4+Data+extra ction

Joanna Briggs Institute Reviewer's Manual (2019). The narrative synthesis of data. Recuperado de https://wiki.joannabriggs.org/display/MANUAL/7.3.6.5.2+The+narr ative+synthesis+of+data

Kim-Spoon, J., Deater-Deckard, K., Holmes, C., Lee, J., Chiu, P., \& King Casas, B. (2016). Behavioral and neural inhibitory control moderates the effects of reward sensitivity on adolescent substance use. Neuropsychologia, 91, 318326. doi:10.1016/j.neuropsychologia.2016.08.028

Kuhn, D. (2006). Do cognitive changes accompany developments in the adolescent brain? Perspectives on Psychological Science, 1, 59-67. doi:10.1111/j.1745-6924.2006.t01-2-.x

Mariño, N., Castro, J., \& Torrado, J. (2012). Funcionamiento ejecutivo en policonsumidores de sustancias psicoactivas. Revista de Psicología Universidad de Antioquia, 4(2), 49-63.

Maturana, H. A. (2011). Consumo de alcohol y drogas en adolescentes. Revista Médica Clínica Las Condes, 22(1), 98-109.

Moher D., Liberati A., Tetzlaff J., \& Altman, D. G. (2009).Preferred Reporting Items for Systematic Reviews and Meta-Analyses: The PRISMA Statement. Annals of Internal Medicine, 151, 264269. doi:10.7326/0003-4819-151-4-200908180-00135 
Moraga, J., \& Cartes-Velásquez, R. (2015). Pautas de cheque, parte II: Quorom y Prisma. Revista chilena de cirugía, 67, 325-330. doi:10.4067/S0718-40262015000300015

Munn, Z., Moola, S., Lisy, K., Riitano, D., \& Tufanaru, C. (2015). Methodological guidance for systematic reviews of observational epidemiological studies reporting prevalence and cumulative incidence data. International journal of evidence-based healthcare, 13, 147-153. doi:10.1097/XEB.0000000000000054

Oliva, A., \& Antolín, L. (2010). Cambios en el cerebro adolescente y conductas agresivas y de asunción de riesgos. Estudios de psicología, 31, 53-66. doi:10.1174/021093910790744563

Peeters, M., Janssen, T., Monshouwer, K., Boendermaker, W., Pronk, T., Wiers, R., \& Vollebergh, W. (2015). Weaknesses in executive functioning predict the initiating of adolescents' alcohol use. Developmental cognitive neuroscience, 16, 139-146. doi:10.1016/j.den.2015.04.003

Pharo, H., Slim, C., Graham, M., Gross, J., \& Hayne, H. (2011). Risky busines: executive function, personality, and reckless behavior during adolescence and emerging adulthood. Behavioral Neuroscience, 125. doi:10.1037/a0025768

Piche, J., Kaylegian, J., Smith, D., \& Hunter, S. (2018). The Relationship between Self-Reported Executive Functioning and Risk-Taking Behavior in Urban Homeless Youth. Behavioral Sciences, 8(1), 6. doi:10.3390/bs8010006

Ramos-Valverde, P., \& Moreno-Rodríguez, C. (2010). Situación actual del consumo de sustancias en los adolescentes españoles: tabaco, alcohol, cannabis y otras drogas ilegales. Salud y Drogas, 10(2), 1336.

Rodríguez, J. C., \& Traverso, C. B. (2012). Sexual behavior in adolescents aged 12 to 17 in Andalusia (Spain). Gaceta sanitaria, 26(6), 519-524. doi:10.1016/j.gaceta.2012.02.005

Romer, D., Betancourt, L. M., Brodsky, N. L., Giannetta, J. M., Yang, W., \& Hurt, H. (2011). Does adolescent risk taking imply weak executive function? A prospective study of relations between working memory performance, impulsivity, and risk taking in early adolescence. Developmental Science, 14, 11191133. doi:10.1111/j.1467-7687.2011.01061.x

Rosabal, G, E., Romero, M, N., Gaquín, R, K., Mérida, H., \& Hernández, M, R. (2015). Conductas de riesgo en los adolescentes. Revista Cubana de Medicina Militar, 44(2), 218-229.

Santa-Cruz, C., \& Rosas, R. (2017). Mapping of Executive Functions / Cartografía de las Funciones Ejecutivas. Estudios de Psicología, 38(2), 284-310. doi:10.1080/02109395.2017.1311459

Tena-Suck, A., Castro-Martínez, G., Marín-Navarrete, R., Gómez-Romero, P., \& Gómez-Martínez, R. (2018). Consumo de sustancias en adolescentes: consideraciones para la práctica médica. Medicina interna de México, 34(2), 264-277. doi:10.24245/mim.v34i2.1595

Toplak, M. E., West, R. F., \& Stanovich, K. E. (2012). Practitioner review: Do performance-based measures and ratings of executive function assess the same construct? Journal of Child Psychology and
Psychiatry, and Allied Disciplines, 54, 131-143. doi:10.1111/jcpp.12001

Torres, G., \& Fiestas, F. (2012). Efectos de la marihuana en la cognición: una revisión desde la perspectiva neurobiológica. Revista Peruana de Medicina Experimental y Salud Pública, 29, 127-134.

Viejo, C., \& Ortega-Ruiz, R. (2017). Cambios y riesgos asociados a la adolescencia. Psychology, Society, \& Education, 7(2), 109-118.

Villegas-Pantoja, M., Alonso-Castillo, M., Benavides-Torres, R., \& GuzmánFacundo, F. (2013). Consumo de alcohol y funciones ejecutivas en adolescentes: una revisión sistemática. Aquichan, 13(2), 234-246.

Yoldi, A. (2015). Las funciones ejecutivas: hacia prácticas educativas que potencien su desarrollo. Páginas de educación, 8(1), 72-98. 